\title{
Re: Antibiotics for Asymptomatic Bacteriuria in Kidney Transplant Recipients
}

\author{
Coussement J, Scemla A, Abramowicz D, Nagler EV, Webster AC \\ Free University of Brussels, Erasmus Hospital, Department of Infectious Diseases and Department of Microbiology, Brussels, Belgium \\ Cochrane Database Syst Rev 2018;2:CD011357. doi: 10.1002/14651858.CD011357.pub2.
}

\section{EDITORIAL COMMENT}

Asymptomatic bacteriuria (AB), defined as bacteriuria without signs or symptoms of urinary tract infection (UTI), is observed up to more than $50 \%$ of kidney transplant recipients. In this Cochrane systematic review, besides the efficacy of treating $A B$ with antibiotics in kidney transplant recipients to prevent symptomatic UTIs, also all-cause mortality and the indirect effects of UTI (acute rejection, graft loss, worsening of graft function) were investigated. For this purpose, The Cochrane Kidney and Transplant Register of Studies (which includes searches of Central, MEDLINE, Embase, conference proceedings, the International Clinical Trials Register, and clinicaltrials.gov) was searched until 1 September 2017 and the authors included two studies (212 participants) comparing antibiotics versus no treatment and had identified three on-going studies. Data were pooled across two studies (total of 212 patients) and overall, the incidence of symptomatic UTI varied between 19\% and 31\% in the groups not treated for AB. Persistence of AB was high regardless of treatment and although the available data were limited, so far, there is still no evidence to suggest antibiotic treatment of $A B$ would improve patient and graft outcomes such as all-cause mortality, graft loss, acute rejection, hospitalization for UTI or graft function. Although the data on adverse reactions were also very limited, however, there seemed to have been no severe adverse event attributable to the antibiotic treatment and non-severe adverse events appeared to be infrequent. Including the results of these ongoing randomised controlled trials in further meta-analyses will help provide more reliable evidence whether to treat $A B$ in kidney transplant recipients.

Yarkın Kamil Yakupoğlu, MD

${ }^{\circ}$ Copyright 2018 by the Association of Urological Surgery / Journal of Urological Surgery published by Galenos Publishing House. 